Dr. Christoph Meier

imc Schweiz AG, Industriestrasse 50a, CH-8304 Wallisellen

christoph.meier@im-c.de

Ansätze für das Controlling betrieblicher Weiterbildung.

Erschienen in: Ehlers, Ulf-Daniel \& Schenkel, Peter (Hrsg.): Bildungscontrolling im E-Learning. Erfolgreiche Strategien und Erfahrungen jenseits des ROI. Berlin, Heidelberg, New York: Springer, 2005, S. 55-64.

\begin{abstract}
Ausgangspunkt für diesen Beitrag ist die Unterscheidung von drei Feldern, die für Weiterbildung und deren Controlling zentral sind: gesellschaftliches Umfeld, betriebliches Funktionsfeld und Lernfeld. In diesem Rahmen werden verschiedene Ansätze für betriebliches (Weiter-) Bildungscontrolling verortet. Kurz beschrieben und charakterisiert werden vier unterschiedliche Ansätze: Kennzahlen-Sammlungen für das Lernfeld, Ansätze für ein Phasen-orientiertes Controlling des Bildungsprozesses, Ansätze der Erfolgsanalyse und der Bestimmung des Return on Invest sowie schließlich auch Strategie-orientierte Ansätze auf der Grundlage der BalancedScorecard-Methodik.
\end{abstract}

Ansätze für das Controlling betrieblicher Weiterbildung .............................. 2

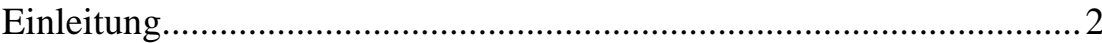

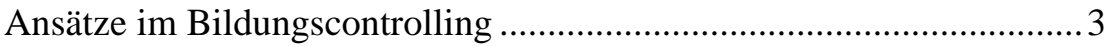

Kennzahlen-Sammlungen für das Lernfeld ....................................... 4

Phasen-orientiertes Controlling des Bildungsprozesses ....................... 5

Erfolgsanalyse und Return on Invest................................................. 7

Strategie-orientiertes Controlling von Weiterbildung mit der

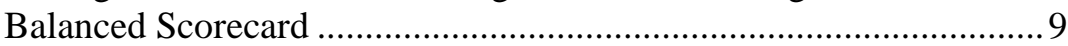

Fazit und Ausblick.............................................................................. 11

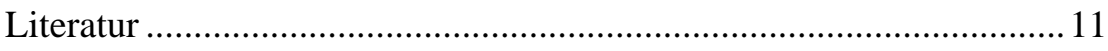

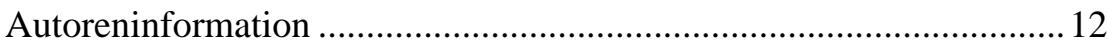




\section{Ansätze für das Controlling betrieblicher Weiterbildung}

\section{Einleitung}

Betriebliche Weiterbildung kann mit verschiedenen Zielen verbunden sein. Die Bandbreite reicht von der Vorbereitung des Einsatzes neuer Arbeitsmittel über strategisch motivierte Bemühungen zur Besetzung zukunftsträchtiger Kompetenzfelder bis hin zu umfassenden Programmen der Personalentwicklung, der Bindung von Mitarbeiterinnen und Mitarbeitern oder der Verbesserung des Unternehmensbildes in der Öffentlichkeit.

Insbesondere dann, wenn Weiterbildung im Hinblick auf ihren kurzund mittelfristigen Beitrag zum Unternehmenserfolg betrieben wird, ist ein betriebliches Weiterbildungscontrolling wichtig. Controlling, so das hier zu Grunde gelegte Verständnis des Begriffs, heißt nicht nur das Herstellen von Transparenz zu Kosten und Ergebnissen. Vielmehr wird Controlling in einem umfassenderen Sinn als Entscheidungsunterstützung und Erfolgssteuerung verstanden. Dazu gehören Tätigkeiten des Planens, Messens, Bewertens und Nachsteuerns [Landsberg 1995, 13f.]. Betriebliches Weiterbildungscontrolling umfasst daher Instrumente und Vorgehensweisen zur ziel- und ergebnisorientierten Planung, Gestaltung und Steuerung der betrieblichen Weiterbildung [Gnahs / Krekel 1999].

Obwohl Unternehmen ihren Mitarbeitern und deren Entwicklung große Bedeutung für den Unternehmenserfolg zuschreiben, fallen Weiterbildungsaktivitäten - vor allem in konjunkturell schwierigen Zeiten - vergleichsweise schnell der Aussicht auf kurzfristige und vergleichsweise einfach zu realisierende Einsparungen zum Opfer. Die für betriebliche Weiterbildung verantwortlichen Personen sehen sich verstärkt mit Fragen danach konfrontiert, ob denn nicht auf diese oder jene Maßnahmen verzichtet werden könne.

Gefordert sind also Verfahren, die es erlauben, den Beitrag von Investitionen in Weiterbildung zum Unternehmenserfolg zu bestimmen und die dafür verfügbaren Ressourcen möglichst effektiv und effizient einzusetzen. 
Zielsetzung dieses Beitrags ist es, einen kurzen Überblick über verschiedene grundlegende Ansätze für das Controlling von Weiterbildung zu vermitteln, auf denen die zahlreichen, in der betrieblichen Praxis vorfindbaren Vorgehensweisen und Verfahren basieren. Dazu gehören Kennzahlen-Sammlungen, Ansätze, die die Betrachtung des Bildungsprozesses in den Mittelpunkt stellen und schließlich auch mehr strategisch orientierte Zugänge auf der Grundlage der Balanced Scorecard Methodik.

\section{Ansätze im Bildungscontrolling}

Zur Orientierung ist es hilfreich, zunächst einmal drei Felder zu unterscheiden, die für Weiterbildung und deren Controlling zentral sind und die wechselseitig aufeinander verweisen. Hierbei handelt es sich zum einen um das Umfeld, in dem Unternehmen sich bewegen und aus dem Anforderungen an Unternehmen herangetragen werden. Dabei kann es sich um Anforderungen von Kunden (Innovation, Qualität, Preis, Zeit) oder auch um Anforderungen auf der Grundlage rechtlicher Bestimmungen und Regelungen (z.B. Ausbildungsquoten oder Vorschriften zur Information von Mitarbeitern, die mit Gefahrstoffen umgehen) handeln. Zum zweiten handelt es sich dabei um das Funktionsfeld, das durch die zentralen betrieblichen Leistungsprozesse konstituiert wird. Aus diesem Feld ergeben sich ebenfalls Anforderungen und Bedarfe für die betriebliche Bildungsarbeit, beispielsweise in Bezug auf die Vermeidung von Fehlern und Ausschuss. Im dritten Feld, dem Lernfeld, werden schließlich die in den beiden zuvor benannten Feldern formulierten Anforderungen und Bedarfe mit Bildungsangeboten beantwortet. Die im Lernfeld vermittelten und von den Mitarbeitern angeeigneten Wissensbestände und Fertigkeiten müssen dann wieder in das Funktionsfeld transferiert werden und dort zur Anwendung kommen. Darüber hinaus müssen Nachweise bezüglich der Erfüllung gesetzlicher Auflagen auch wieder in das Umfeld kommuniziert werden (vgl. Abb. 1). 


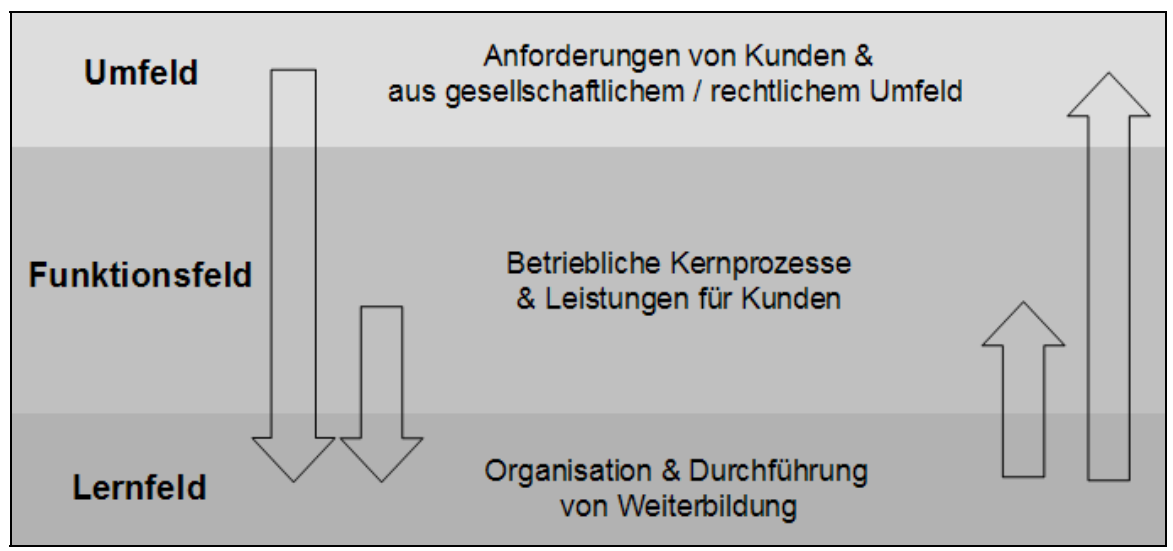

Abb. 1. Umfeld, Funktionsfeld und Lernfeld

Die verschiedenen, in den nachfolgenden Abschnitten kurz vorgestellten Ansätze für betriebliches (Weiter-)Bildungscontrolling unterscheiden sich in der Art und Weise, wie der Zusammenhang zwischen Lern- und Funktionsfeld in den Blick genommen wird.

\section{Kennzahlen-Sammlungen für das Lernfeld}

Kennzahlen und Kennzahlen-Sammlungen als Grundlage für Bewertungen und Vergleiche sind ein grundlegender Zugang für Controlling-Aktivitäten in verschiedensten Bereichen. Im Mittelpunkt des von [Schulte 1995] vorgeschlagenen Systems von Kenngrößen für Bildungscontrolling steht das Herstellen von Transparenz zu verschiedenen Aspekten des Lernfelds. Schulte entwickelt hierzu eine Sammlung von Kenngrößen, die sich auf die Inhalte, Teilnehmer, Methoden, Kosten und schließlich auch die Erfolge von Weiterbildung beziehen (vgl. Abb. 2). 


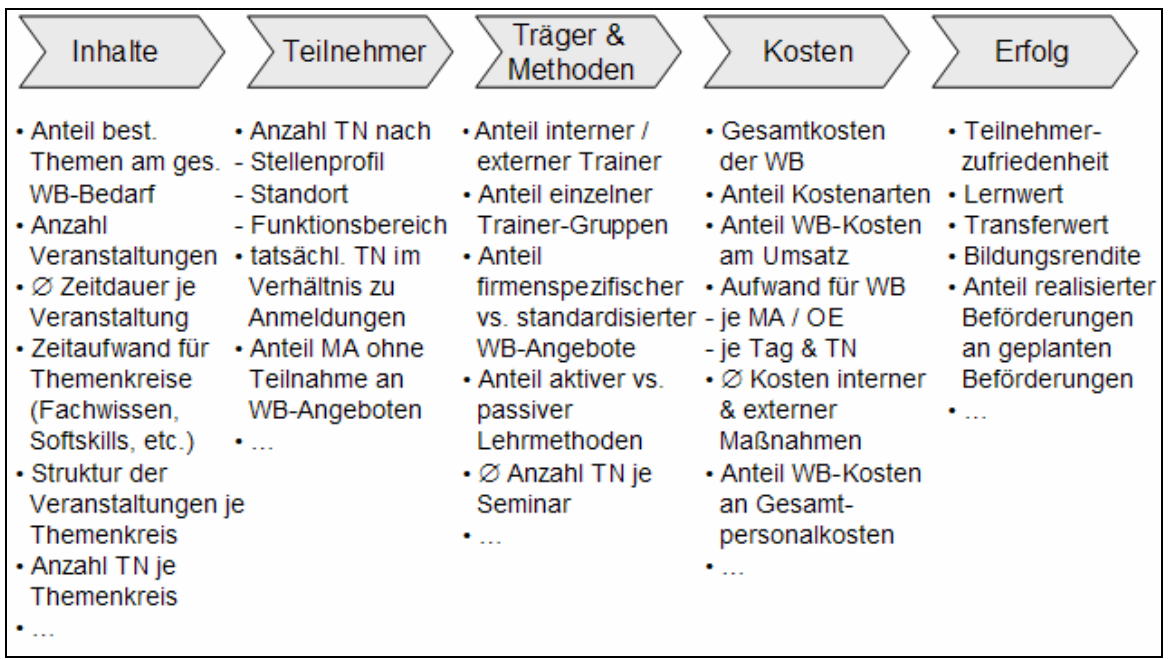

Abb. 2. Kennzahlen für das Weiterbildungscontrolling [Schulte 1995]

Kennzahlen-orientierte Ansätze im Weiterbildungscontrolling sorgen für Orientierung und ermöglichen eine gezielte Datenerhebung. Allerdings stellt sich immer auch die Frage, auf Grund welcher Überlegungen diese oder jene Kenngröße herangezogen wird. Die Beantwortung relevanter Fragen für das Weiterbildungscontrolling (,Werden die wichtigsten Zielgruppen erreicht?“; „Werden die besten Anbieter ausgewählt?“; „Werden die Ziele der Maßnahmen erreicht?“ etc.) steht und fällt mit der sachgerechten Auswahl der Kenngrößen. Unter Umständen besteht daher die Gefahr, dass unübersichtliche und letztlich auch willkürliche Sammlungen von Kenngrößen entwickelt werden [Gerlich 1999, S. 22].

\section{Phasen-orientiertes Controlling des Bildungsprozesses}

In der oben vorgestellten Kennzahlen-Sammlung wird der Prozess der betrieblichen Weiterbildung nicht genau in den Blick genommen. Dabei ist die Art und Weise, wie Weiterbildungsangebote zustande kommen und umgesetzt werden, ein wichtiger Erfolgsfaktor für betriebliche Bildungsarbeit. Phasen-orientierte Ansätze des Bildungscontrollings nehmen genau diesen Verlauf in den Blick: von der Bedarfsermittlung über die Planung / Konzeption, die Umsetzung / Realisierung, die Durchführung, die Transferunterstützung bis hin zur Erfolgsanalyse [vgl. Hummel 2001, S. 25-27; Gerlich 1999, 35-40]. 
Betrachtet man den Bildungsprozess oder Bildungszyklus genau, dann werden nicht nur die im Verlauf dieser Phasen generierten Aufwände und Kosten sichtbar und zuordnungsfähig. Auch die Instrumente und Verfahren zur Bewältigung der verschiedenen Aufgaben werden für die kritische Begutachtung und die Frage nach Möglichkeiten der Optimierung zugänglich: „Wie wird der Soll-Ist-Vergleich von Rollenprofilen und Mitarbeiterkompetenzen bewerkstelligt und wie lange dauert das?“ „Können einzelnen Lernern, auf der Grundlage von Skill-Gap-Analysen, automatisch passende online-Lernangebote, beispielsweise zu Sprachen oder Projektmanagement, vorgeschlagen werden oder muss dies manuell geschehen?“ „Sind die Kriterien, auf deren Grundlage externe Bildungsanbieter ausgewählt werden, noch aktuell?“ „Wie aufwändig ist es, eine Lernerfolgskontrolle zu einem Kurs zu erstellen, durchzuführen und auszuwerten?“

Das Rahmenkonzept für Bildungscontrolling von [Gerlach 1999], um hier nur ein Beispiel anzuführen, berücksichtigt nicht nur den Phasenverlauf betrieblicher Weiterbildung, sondern darüber hinaus auch die Anbindung an das betriebliche Funktionsfeld mit den dort formulierten Zielen (Abb. 3).

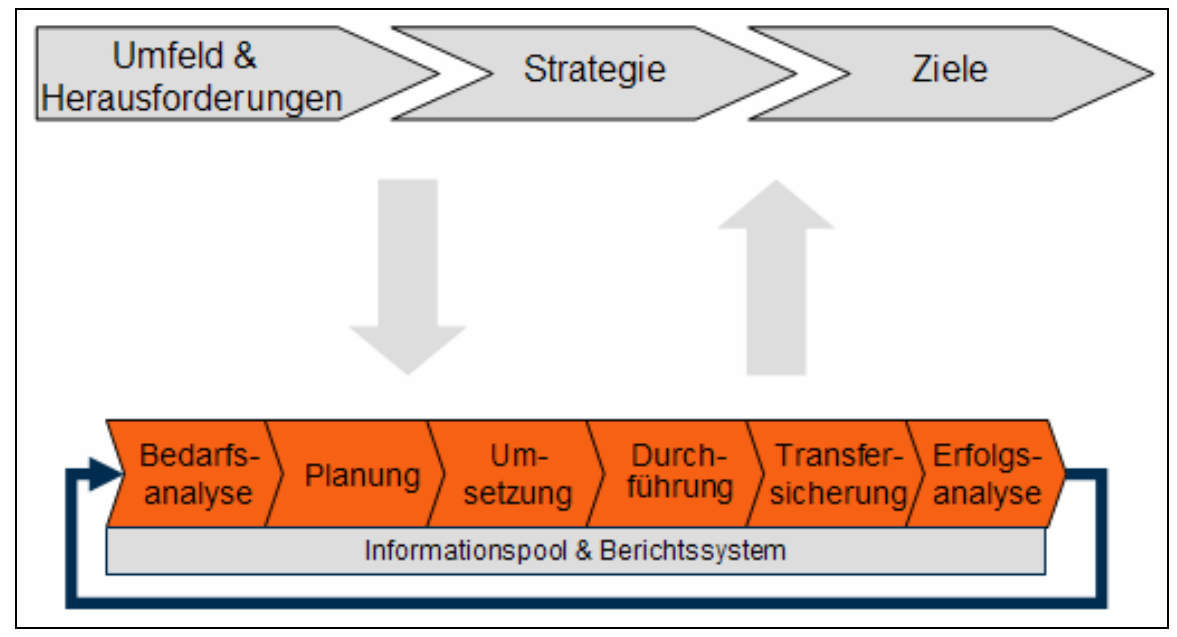

Abb. 3. Phasenorientiertes Rahmenkonzept für Bildungscontrolling in Anlehnung an [Gerlich 1999] 


\section{Erfolgsanalyse und Return on Invest}

In der letzten Zeit ist im Zusammenhang mit der Diskussion um Bildungscontrolling immer wieder der Return on Invest von Bildungsmaßnahmen als die wichtigste Kenngröße bezeichnet worden. Ist die Ermittlung dieser gelegentlich als der „heilige Gral der Bildungscontroller“ bezeichneten Kenngröße realistisch? Wie kann der mit dem Erfolg von Bildungsmaßnahmen verbundene Mehrwert bestimmt werden?

Für die Evaluation betrieblicher Weiterbildung hat [Kirkpartrick 1996, ursprünglich 1959] eine mittlerweile klassische Systematik geliefert. Er unterscheidet vier verschiedene Ebenen, auf denen der Erfolg von Weiterbildung bestimmt werden kann:

1. Akzeptanz für das Lernangebot,

2. Lernerfolg,

3. Verhalten im Arbeitsfeld (Transfer) und schließlich

4. Ergebnisse im Anwendungsfeld (Geschäftsergebnis).

Diese Systematik ist vielfach aufgegriffen und auch erweitert worden. So ergänzt beispielsweise [Schenkel 2000] eine untere Ebene, auf der ein Bildungsangebot von Experten auf definierte Qualitätsmerkmale hin geprüft wird. [Preisner 2003] schlägt vor, Lernerfolg nicht nur auf die Aneignung von Inhalten zu beziehen, sondern auch auf die Entwicklung der Fähigkeit zu weiterem Lernen (Lernkompetenz). Darüber hinaus plädiert er dafür, auf der Ebene 4 (Ergebnisse im Anwendungsfeld) nicht nur nach dem betrieblichen, sondern auch nach dem persönlichen Erfolg zu fragen.

Die Systematik von Kirkpatrick ist auch der Ausgangspunkt für [Phillips 1997] und den von ihm formulierten „ROI-Process“. Auch Phillips schlägt eine weitere Ebene der Evaluation vor: den Return-on-invest von Weiterbildungsmaßnahmen. Darüber hinaus formuliert er detailliert ein Vorgehensmodell zur Durchführung solcher Analysen.

Ausgangspunkt für Phillips sind die Geschäftsmöglichkeiten und die mit ihnen verbundenen Anforderungen an die Qualifikation der Mitarbeiter. Sofern diese durch Weiterbildung (und nicht durch das Rekrutieren neuer Mitarbeiter) erfüllt werden sollen, müssen zunächst die Lernbedarfe und die präferierten Lernformen der Zielgruppen bestimmt werden (vgl. Abb. 4). 


\begin{tabular}{|c|c|c|c|c|c|c|c|}
\hline \multicolumn{3}{|c|}{ Geschäftsmöglichkeit } & $\begin{array}{l}\text { Ebene } 5 \\
\text { ROI / ROE }\end{array}$ & \multicolumn{3}{|c|}{ Bestimmung ROI } & \\
\hline \multicolumn{2}{|c|}{$\begin{array}{c}\text { Geschäfts- } \\
\text { anforderungen }\end{array}$} & \multirow{3}{*}{ 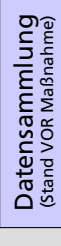 } & $\begin{array}{c}\text { Ebene } 4 \\
\text { Geschäftsergebnis }\end{array}$ & \multirow{3}{*}{ 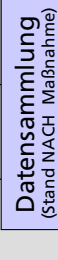 } & \multirow{4}{*}{ 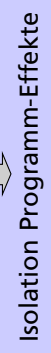 } & \multirow{4}{*}{ 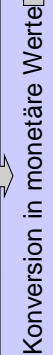 } & \multirow{4}{*}{ 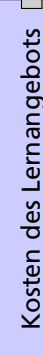 } \\
\hline \multicolumn{2}{|c|}{$\begin{array}{l}\text { Qualifikations- } \\
\text { anforderungen } \\
\text { am Arbeitsplatz }\end{array}$} & & $\begin{array}{c}\text { Ebene } 3 \\
\text { Arbeitshandeln }\end{array}$ & & & & \\
\hline & Lerr & & $\begin{array}{c}\text { Ebene } 2 \\
\text { Lernerfolg }\end{array}$ & & & & \\
\hline & $\begin{array}{l}\text { Präf } \\
\text { Lern }\end{array}$ & & $\begin{array}{c}\text { Ebene } 1 \\
\text { Akzeptanz }\end{array}$ & & & & \\
\hline $\begin{array}{l}\text { Lösung ohne PE } \\
\text { (Recruiting etc.) }\end{array}$ & $\begin{array}{r}\text { Eval } \\
\text { stra } \\
-p l\end{array}$ & & $\begin{array}{l}\text { Entwicklung \& } \\
\text { Durchführung } \\
\text { WB-Maßnahme }\end{array}$ & & & & \\
\hline
\end{tabular}

Abb. 4. Der ROI-Process nach Phillips

Vor der Durchführung von Weiterbildungsmaßnahmen sollten systematisch Daten auf den Ebenen 3 und 4 (Arbeitshandeln und Geschäftsergebnisse) erhoben werden, da nur auf diese Weise Vergleiche mit dem Stand danach möglich sind. Methodisch anspruchsvoll sind dann die erforderliche Isolation der Effekte der Weiterbildungsmaßnahmen und deren Konversion in monetäre Werte. Für die Isolation der Weiterbildungseffekte können beispielsweise Verfahren der Trendanalyse oder auch Einschätzung von Veränderungen durch die Beteiligten eingesetzt werden. Klassische Kenngrößen sind etwa Produktionsziffern, Zeiten, Kosten oder Qualitätsgrößen. Zur Konversion der Ergebnisse in monetäre Größen können beispielsweise Deckungsbeitragswerte, Erfahrungswerte zu den Kosten von Qualitätsverbesserungen, Kosten für Mitarbeiter oder auch Einschätzungen durch die Beteiligten herangezogen werden [Phillips 1997, S. 87131]. Erst auf der Grundlage dieser Schritte wird dann eine Berechnung des Return on Invest möglich.

So attraktiv das Bemühen um eine monetäre Bewertung der Auswirkungen von Weiterbildung ist, so klar sind auch die damit verbundenen Anforderungen. Eine ROI-Kalkulation kann nur dort erfolgreich sein, wo auf quantifizierbare und anschließend monetarisierbare Größen rekurriert werden kann. Für sogenannte „harte“ Kenngrößen wie etwa

- die Zahl produzierter oder verkaufter Einheiten,

- die erforderliche Zeit für die Bearbeitung einer Anfrage oder für das Reparieren eines Gegenstands,

- die Kosten für das Erbringen einer bestimmten Leistung, 
ist dies deutlich einfacher als für sogenannte „weiche“ Kenngrößen, beispielsweise

- die Motivation von Mitarbeitern und deren Bindung an das Unternehmen,

- die Kundenorientierung von Mitarbeitern,

- die Kreativität von Mitarbeitern.

Verkaufstrainings sind nicht zuletzt deshalb so beliebte Beispiele für die Berechnung des ROI von Weiterbildung, da hier eine solche Kalkulation vergleichsweise einfach ist. Bei der Berechnung des Return on Invest für ein Training von Projektleitern wird dies aber schon deutlich schwieriger. Dies gilt erst recht für die Berechnung des ROI von längerfristig angelegten Programmen der Personalentwicklung, beispielsweise für den Führungskräfte-Nachwuchs.

\section{Strategie-orientiertes Controlling von Weiterbildung mit der Balanced Scorecard}

Die Frage, wie die Auswahl von Kenngrößen für das Weiterbildungscontrolling begründet ist und ob diese der Situation des Unternehmens angemessen sind, wurde bereits aufgeworfen. Wenn es, beispielsweise, erklärtes Ziel eines Unternehmens ist, seine Mitarbeiter für selbst gesteuertes Lernen zu gewinnen und zu qualifizieren, um dadurch langfristig flexibler agieren zu können, dann ist die Zahl der pro Jahr durchgeführten Präsenzschulungen keine sinnvolle Kenngröße für das betriebliche Bildungscontrolling. Und auch die Bestimmung des Return on Invest für Trainings muss dann dieses strategische Ziel berücksichtigen.

Die von Kaplan / Norton [1997] entwickelte Balanced ScorecardMethodik ist ein Instrument zur Steuerung und Kontrolle der Umsetzung einer Unternehmensstrategie in operatives Handeln. Sie rückt neben den traditionell im Controlling in den Mittelpunkt gestellten finanziellen Kenngrößen verschiedene weitere Felder von Erfolgsfaktoren in den Blick. Dazu gehören die Felder „Lernen \& Entwicklung“ mit Fokus auf Humanressourcen und deren Entwicklung, „interne Prozesse“ wie beispielsweise Betriebsprozesse und schließlich auch kundenbezogene Kenngrößen wie beispielsweise Kundenzufriedenheit, Kundentreue oder Kundenrentabilität.

Die Balanced Scorecard dient dabei zugleich auch als strategisches Managementsystem. Der damit verbundene Prozess beinhaltet vier Phasen [Kaplan / Norton 1997, S. 10-15]: 
- die Formulierung einer Vision und eines gemeinsamen Zielsystems;

- die Kommunikation der Ziele innerhalb des Unternehmens und deren Verknüpfung mit Kennzahlen und Anreizsystemen;

- das Operationalisieren der Ziele auf untergeordneten Ebenen; und schließlich

- den Review der Ergebnisse und ein Feedback dazu, ob sich die Strategie bewährt hat.

Die Balanced Scorecard-Methodik beinhaltet nicht nur die Entwicklung einer ausgeklügelten Sammlung von Kenngrößen. Darüber hinaus wird den Wirkungszusammenhängen innerhalb und zwischen den vier Feldern große Aufmerksamkeit gewidmet. Ziel ist es, eine Sammlung von wenigen (in der Regel etwa 20) Erfolgsfaktoren und relevanten Kennzahlen zu entwickeln, die eine möglichst geschlossene Wirkkette darstellen und die damit Ansatzpunkte für die Steuerung und Erfolgskontrolle bei der Umsetzung der Unternehmensstrategie markieren (vgl. [Kaplan / Norton 1997] und Abb. 5).

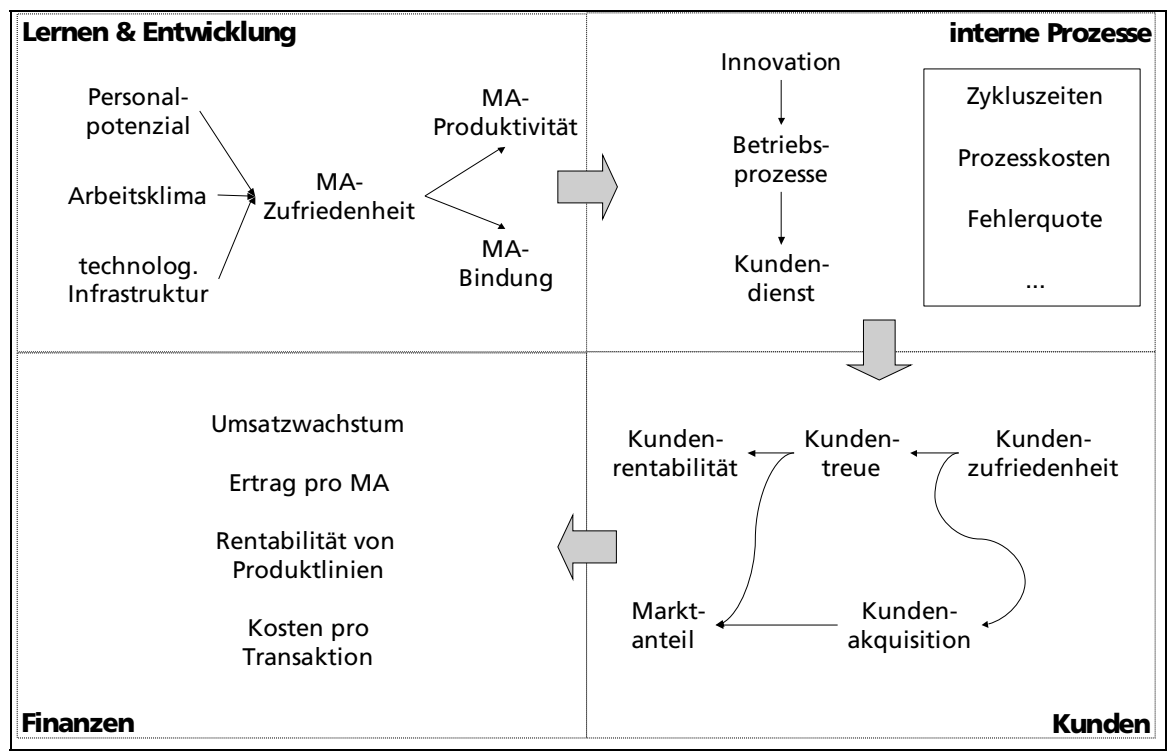

Abb. 5. : Perspektiven, Erfolgsfaktoren und Wirkungszusammenhänge der BSC

Die Balanced Scorecard-Methodik hat den Bereich Personalentwicklung und Weiterbildung innerhalb von Unternehmen strategisch aufwertet und das Potenzial dieses Ansatzes für die Evaluation von Weiterbildung und Personalentwicklung wird diskutiert [Greiling 2001; Diensberg 2001]. Da- 
bei wird aber unter anderem auch darauf verwiesen, dass viele der Unternehmen, die mit dieser Methodik arbeiten, das Feld „Lernen \& Entwicklung“ unberücksichtigt lassen und dass insbesondere das Erstellen von Ursache-Wirkungs-Ketten und die Beschränkung auf wenige entscheidende Kenngrößen für die Anwender eine echte Herausforderung darstellt.

\section{Fazit und Ausblick}

Wie der kurze Überblick im vorangegangenen Kapitel gezeigt hat, sind verschiedene Ansätze für das betriebliches Weiterbildungscontrolling formuliert worden, die jeweils unterschiedliche Aspekte fokussieren: die Sammlung von Kenngrößen, die Betrachtung des Bildungsprozesses, die Bestimmung des Weiterbildungserfolgs und insbesondere des Return-oninvest und schließlich ein stärker Strategie-orientierter Zugang auf der Grundlage der Balanced Scorecard-Methodik.

Die vorgestellten Ansätze finden sich in der einen oder anderen Form in verschiedenen Beiträgen in diesem Band wieder. So wird etwa der Ansatz der Balanced Scorecard in einem separaten Kapitel behandelt (siehe den Beitrag von Back, Kap. 10) vertieft behandelt. Eine kurze Skizze für eine Synthese der in diesem Beitrag vorgestellten Ansätze zu einem adaptierten Balanced-Scorecard-Ansatz findet sich im Beitrag von Meier (Kap. 11), ebenfalls in diesem Band.

\section{Literatur}

DIENSBERG, Christoph: Balanced Scorecard - kritische Anmerkungen für die Bildungs- und Personalarbeit. In: Diensberg, C.; Krekel, S.; Schobert, B. (Hrsg.): Balanced Scorecard und House of Quality. Impulse für die Evaluation in Weiterbildung und Personalentwicklung. Wissenschaftliche Diskussionspapiere Nr. 53, Bundesinstitut für Berufsbildung, Bonn, 2001, S. 21-38.

GERLICH, Petra: Controlling von Bildung, Evaluation oder BildungsControlling? München / Mering: Hampp, 1999.

GNAHS, Dieter und KREKEL, Elisabeth M.: Betriebliches Bildungscontrolling in Theorie und Praxis: Begriffsabgrenzung und Forschungsstand. In: Krekel, E.M. und Seusing, B. (Hrsg.): Bildungscontrolling - ein Konzept zur Optimierung der betrieblichen Weiterbildung, hrsg. vom Bundesinstitut für Berufsbildung. Bielefeld, Bertelsmann 1999, S. 13-33.

GREILING, Michael: Die Balanced Scorecard. In: Diensberg C.; Krekel, E.M.; Schobert, B. (Hrsg.): Balanced Scorecard und House of Quality. Impulse für die Evaluation in Weiterbildung und Personalentwicklung. Wissenschaftliche 
Diskussionspapiere Nr. 53, Bundesinstitut für Berufsbildung, Bonn, 2001, S. 9-20.

HUMMEL, Thomas R.: Erfolgreiches Bildungscontrolling. Zweite Auflage. Heidelberg: Sauer, 2001.

KAPLAN, Robert S. und NORTON, David P.: Balanced Scorecard. Strategien erfolgreich umsetzen. Stuttgart: Schäffer-Poeschl, 1997.

KIRKPATRICK, Donald: Evaluating training programs: The four levels. San Francisco: Berrett-Koehler, 1996.

LANDSBERG, Georg von: Bildungs-Controlling: What is likely to go wrong? In: Bildungscontrolling. 2., überarbeitete Auflage, hrsg. von G. von Landsberg \& R. Weiß. Stuttgart: Schaeffer-Poeschel 1995, S. 11-33.

PHILLIPS, Jack J.: Return on investment in training and performance improvement programs. Houston: Gulf, 1997.

PREISNER, Klaus: Bildungscontrolling. Individuumorientierte Konzeption zur verbesserten Koordination des betrieblichen Weiterbildungssystems. Dissertation, Universität der Bundeswehr, München, Fakultät für Wirtschafts- und Organisationswissenschaften, 2003.

SCHENKEL, Peter: Ebenen und Prozesse der Evaluation. In: Tergan, S.O. u.a. (Hrsg.): Qualitätsbeurteilung multimedialer Lern- und Informationssysteme: Evaluationsmethoden auf dem Prüfstand. Nürnberg, BW-Verlag, 2000, S. 5274.

SCHULTE, Christof: Kennzahlengestütztes Weiterbildungs-Controlling als Voraussetzung für den Weiterbildungserfolg. In: Bildungscontrolling. 2., überarbeitete Auflage, hrsg. von G. von Landsberg \& R. Weiß. Stuttgart: SchaefferPoeschel 1995, S. 265-281.

\section{Autoreninformation}

Dr. Christoph Meier ist seit dem Jahr 2004 bei der imc AG als Senior Consultant beschäftigt und leitet das Competence Center Bildungscontrolling. Dr. Meier arbeitet seit fast 10 Jahren im Feld medial vermitteltes Arbeiten und Lernen zunächst in Grundlagen-orientierten Forschungsprojekten, dann in Projekten der angewandten Forschung und heute als Berater. Nach seiner Tätigkeit an der Universität Giessen war er am Fraunhofer-Institut für Arbeitswirtschaft und Organisation (Stuttgart) beschäftigt, bevor er zu imc wechselte. Seine zahlreichen Veröffentlichungen befassen sich mit der Analyse von Kommunikationsprozessen, Telekooperation, medial gestütztem Lernen (E-Learning) und Bildungscontrolling. 